\title{
Community treatment orders: an analysis of the first five years of use in England
}

\author{
Liam Trevithick, Jane Carlile, Sunil Nodiyal and Patrick Keown
}

\section{Background}

Community treatment orders (CTOs) were introduced in England in 2008.

\section{Aims}

To measure the rate of CTO use in England during the first 5 years following introduction.

\section{Method}

The number of involuntary detentions and CTOs in National Health Service (NHS) hospital trusts was collected between 2009 and 2014. Rates of CTO use and the ratio of CTOs to detentions on admission were calculated, and how these varied between trusts.

\section{Results}

The number of new CTOs each year ranged between 3834 and 4647. The number subject to a CTO per 100000 population increased from 6.4 in 2009/10 to 10.0 in 2013/14. There was variation between NHS trusts in the use of CTOs when compared with the number of involuntary detentions

\section{Conclusions}

The number of patients on CTOs increased year on year. Those on forensic sections were more likely to be discharged on a CTO than those on civil sections. There was considerable variation in the pattern of use between hospitals.

\section{Declaration of interest}

None.

\section{Copyright and usage}

(c) The Royal College of Psychiatrists 2018
In November 2008, involuntary community treatment was introduced in England and Wales in the form of community treatment orders (CTOs). Involuntary community treatment can be conceived of as part of the move from primarily hospital-based care to the current situation in many countries where the majority of psychiatric care occurs in the community. ${ }^{1}$ Involuntary community treatment has not been introduced in all jurisdictions across the world and controversy remains because of the lack of clear evidence of effectiveness. ${ }^{2}$ Proponents of CTOs claim that they promote patient engagement with services and treatment, while reducing the need for in-patient stays. ${ }^{3-5}$ Opponents point to the lack of empirical evidence to support the effectiveness of CTOs from Western countries where similar provisions are already in place ${ }^{6,7}$ and also to the infringement of civil liberties. ${ }^{8,9}$

In England and Wales there was a long debate about extending involuntary community treatment beyond that currently available. ${ }^{10}$ A working party in the 1990 s identified a group of individuals who might benefit: those with a history of involuntary in-patient treatment who respond to treatment but disengage from treatment on discharge and are readmitted again on an involuntary basis. ${ }^{11}$ It was estimated that between 3000 and 5000 patients nationally might meet the criteria for such an order, but this has not yet been confirmed and more recently it was estimated there could be as few as between 400 and 600 CTOs per year. ${ }^{12}$ Since then a randomised controlled trial of discharges on CTOs in England found no evidence of effectiveness at 12 months, ${ }^{13}$ or subsequently at 36 month follow-up. ${ }^{14}$ A recent Cochrane review of involuntary community treatment showed no difference in service use, social functioning, mental state or quality of life. ${ }^{15}$ Despite this lack of evidence, the perception is that CTOs have quickly become an established part of mental health services. The first aim of this paper is to quantify the use of CTOs in England in the first 5 years following their introduction.

Where involuntary community treatment has been introduced, it has taken several different forms. In some areas the powers are independent of or an alternative to involuntary in-patient treatment. $^{2}$ In England and Wales, CTOs can only be used when a patient is being discharged from a period of involuntary inpatient treatment. The main power is of recall to hospital, which removes the need for a new detention on admission to hospital. The second aim of this paper is to investigate the relationship between involuntary treatment in hospital under the Mental Health Act and the use of CTOs on discharge from hospital.

Recent evidence demonstrates the higher rates of involuntary detention to hospital in urban areas compared with more rural areas across England. ${ }^{16}$ Additionally there is significant variation in the extent to which involuntary community treatment is used both between and within different countries. ${ }^{15}$ For example in Australia rates of new CTOs started each year ranged from 30.2 per 100000 population in Tasmania to 98.8 per 100000 in Victoria. ${ }^{17}$ The third aim of this paper is to describe variation in the use of CTOs between National Health Service (NHS) trusts; and in particular whether there are differences in the use between urban trusts and non-urban trusts.

\section{Method}

The data used were obtained from the Health and Social Care Information Centre online Omnibus KP90 collection. This is publicly available data. The number of new CTOs and the number of individuals subject to a CTO on the 31 March each year were recorded for the years 2009-10 to 2013-14. Rates were calculated per 100000 of the total population using data from the Office for National Statistics. ${ }^{18}$ The type of detention in hospital preceding the CTO was divided into two groups: civil detentions (section 3); and forensic detentions (sections 37 and 47). Forensic sections are hospital orders that are instigated by a court of law or from prisons. Patients detained on forensic sections can be detained with or without restriction orders. The Ministry of Justice is involved in decisions about leave and discharge for all patients on restriction orders. Only those detained without restriction orders are eligible for discharge on a CTO. Patients who were detained following the revocation of a CTO were included in the 'all detentions' 
figures. Those recalled to hospital under a CTO but then discharged prior to revocation (within $72 \mathrm{~h}$ ) were not included.

The number of new CTOs each year was compared with the total number of involuntary detentions in hospital and the number of involuntary detentions on admission in the same year. This allowed the calculation of a ratio, the purpose of which was to give a proxy measure of the proportion of detained patients who were discharge on CTOs compared with discharge on a voluntary basis. The same process was repeated separately for civil detentions and forensic detentions.

The ratio of new CTOs to involuntary detention on admission to hospital was also calculated as CTOs were specifically intended for patients who are repeatedly discharged and then readmitted under the Mental Health Act. This information (the number of CTOs and the number of involuntary detention on admission) was also available for each NHS hospital trust in England. Hospital trusts that provided data for a minimum of 3 out of the 5 years were included. To increase the reliability of the analysis only those hospitals that had more than ten formal admissions in each year were included. Mental health hospital trusts were then compared with each other. Three data points were removed from analysis because of suspected data errors (Appendix).

\section{Results}

\section{The annual number and rate of Стоs and the relationship with the annual number of detentions in hospital}

There was a total of 21242 new CTOs in the first 5 years of their use in England between 2009/10 and 2013/14. This ranged from 3834 to
4647 each year (Table 1). Most $(93 \%, n=19834)$ CTOs followed a civil involuntary detention in hospital; $4 \%$ (862) followed on from forensic involuntary detention in hospital; and for 3\% (546) this was not specified.

The average yearly rate of new CTO use was 8.0 (s.d. $=0.5$ ) per 100000 , with a $5 \%$ increase in the rate during this time from 7.9 to 8.2. The rate of CTO use following civil detention was 7.5 (s.d. $=0.5$ ) per 100000 and this increased by $6 \%$ from 7.2 to 7.6 . The rate of CTO use following forensic detentions was lower at 0.3 (s.d. $=0.1$ ) per 100000 , but increased by $42 \%$ over the 5 -year period from 0.2 to 0.3 (Table 1).

The number of individuals subject to a CTO on the 31 of March each year increased during the study period. At the end of each year there were more patients who were subject to CTOs than there were a year previously. This can be considered the prevalence rate of CTOs and this increased by $56 \%$ from 6.4 per 100000 in 2010 to 10.0 per 100000 in 2014 .

\section{The ratio of СТOs to involuntary in-patient treatment}

The average annual number of new CTOs in England was 4248 (s.d. $=311)$. This compared with an average of 49032 (s.d. $=2843$ ) involuntary detentions, of which 31534 (s.d. $=2080$ ) were detentions that occurred at the time of admission. This meant that there was one discharge on a CTO for every 11.6 involuntary detentions (95\% CI 10.9-12.2), a ratio of 0.09 (95\% CI 0.08-0.09); and one discharge on CTO for every 7.4 detentions on admission (95\% CI $7.0-7.9$ ), a ratio of 0.14 (95\% CI $0.13-0.14$ ). Table 2 shows that although the number of CTOs increased overall, so did the number of involuntary detentions in hospital. As a result, the ratio of new CTOs to involuntary detentions remained relatively stable in the first 5 years.

\begin{tabular}{|c|c|c|c|c|c|c|}
\hline Year & $2009 / 2010$ & $2010 / 2011$ & $2011 / 2012$ & $2012 / 2013$ & $2013 / 2014$ & $\%$ change \\
\hline Number of new CTOs & 4107 & 3834 & 4220 & 4647 & 4434 & +8 \\
\hline Number after civil detention & 3736 & 3606 & 4004 & 4389 & 4099 & +10 \\
\hline Number after forensic detention & 127 & 164 & 185 & 203 & 184 & +45 \\
\hline Number individuals on CTOs on 31 March & 3325 & 4291 & 4764 & 5218 & 5365 & +61 \\
\hline Population of England (1000 s) & 52196 & 52642 & 53107 & 53494 & 53866 & +3 \\
\hline Incidence of new CTOs per 100000 & 7.9 & 7.3 & 8.0 & 8.7 & 8.2 & +5 \\
\hline Incidence rate following civil detention & 7.2 & 6.9 & 7.5 & 8.2 & 7.6 & +6 \\
\hline Incidence rate following forensic detention & 0.2 & 0.3 & 0.4 & 0.4 & 0.3 & +42 \\
\hline Number of individuals subject to CTOs on 31 March per 100000 population & 6.4 & 8.2 & 9.0 & 9.8 & 10.0 & +56 \\
\hline
\end{tabular}

Table 2 The number of involuntary detentions in hospital under The Mental Health Act, detentions on admission to hospital and discharges on com-
munity treatment orders (CTOS), 2008/09 to 2013/14 in England
Year




\begin{tabular}{|c|c|c|c|c|c|}
\hline Year & $2009 / 10$ & $2010 / 11$ & $2011 / 12$ & $2012 / 13$ & $2013 / 14$ \\
\hline NHS trusts, $n$ & 67 & 69 & 68 & 66 & 69 \\
\hline Number of discharges on CTOs, mean (s.d.) & $62.8(51.2)$ & $59.0(51.2)$ & $56.6(51.4)$ & $55.5(51.4)$ & $61.2(51.5)$ \\
\hline Number of detentions on admission, mean (s.d.) & $424.9(310.6)$ & $408.5(310.7)$ & $374.9(312.1)$ & $377.0(312.2)$ & $438.4(312.3)$ \\
\hline New CTOs/detentions on admission, ratio $(95 \% \mathrm{Cl})$ & $0.13(0.11-0.15)$ & $0.13(0.11-0.15)$ & $0.14(0.12-0.16)$ & $0.14(0.12-0.16)$ & $0.12(0.10-0.14)$ \\
\hline Range for ratio & $0.00-0.43$ & $0.00-0.46$ & $0.00-0.49$ & $0.00-0.36$ & $0.00-0.31$ \\
\hline
\end{tabular}

The likelihood of being discharged on a CTO was higher for those discharged following forensic detentions without restriction orders: there was one discharge on a CTO for every 5.9 civil detentions (95\% CI 5.4-6.4); a ratio of 0.17 (95\% CI 0.16-0.18) that varied little between the years. In contrast there was one discharge under a CTO for every 2.7 forensic detentions (95\% CI 1.6-3.8); a ratio of 0.37 (95\% CI 0.26-0.62). Table 2 shows that this ratio doubled in the 5 -year period. This was because of a combination of more CTOs and fewer forensic detentions without restriction orders. The number of forensic detentions without restrictions also fell but to a lesser degree ( $14 \%$ reduction compared with $29 \%$ reduction).

\section{The variation between NHS hospital trusts in the ratio of CTOs and detention on admission to hospital}

On average 124 (range 120-130) NHS trusts provided information each year on the number of new CTOs and the number of detentions on admission to hospital. In total over the 5 years 171 NHS hospital trusts provided this information. Of these 82 delivered acute medical/surgical/paediatric/maternity care; 63 delivered mental health and intellectual disability services (in the UK known as learning disability services); and 26 delivered community hospital and primary care services.

In total, 50 of the 171 NHS hospital trusts were excluded as they only provided information for 2 of the 5 years. Another 47 were excluded as they had less than 10 detentions on admission in a year. This left 74 NHS trusts, which included 57 of the 63 NHS trusts that provided mental health and learning disability services. Of the 157674 detentions on admission in hospital in England in the 5 years of the study 137273 (87\%) occurred in these 74 trusts.

Table 3 shows that there was a consistent ratio between the number of detentions on admission in NHS hospital trusts each year and the number of discharges on CTOs: one new CTO for every 7.7 detentions on admission; or a ratio of 0.13 .

However, there was a large range in this ratio. This was partly explained by the type of health trust. Some NHS trusts that mainly provided in-patient medical and surgical services had a number of detentions on admission, but rarely discharged patients on CTOs. As can been seen in Fig. 1, these trusts had very low ratios of new CTOs to detention at time of admission $(n=13$. Mean ratio 0.00 , s.d. $=0.01$ ). Trusts with in-patient services that were mainly for mental health and learning disability had much higher rates of discharge on CTOs $(n=57$, mean ratio 0.15 , s.d. $=0.06)$. This ratio ranged from 0.06 - one new CTO for every 17 detentions on admission, to a ratio of 0.36 - one new CTO for every 3 detentions on admission.

There were 13 NHS trusts located in five major urban areas (London, Birmingham, Manchester, Liverpool and Leeds) in England that tended to have higher ratios of discharges on CTOs than detentions on admission. These NHS hospital trusts are

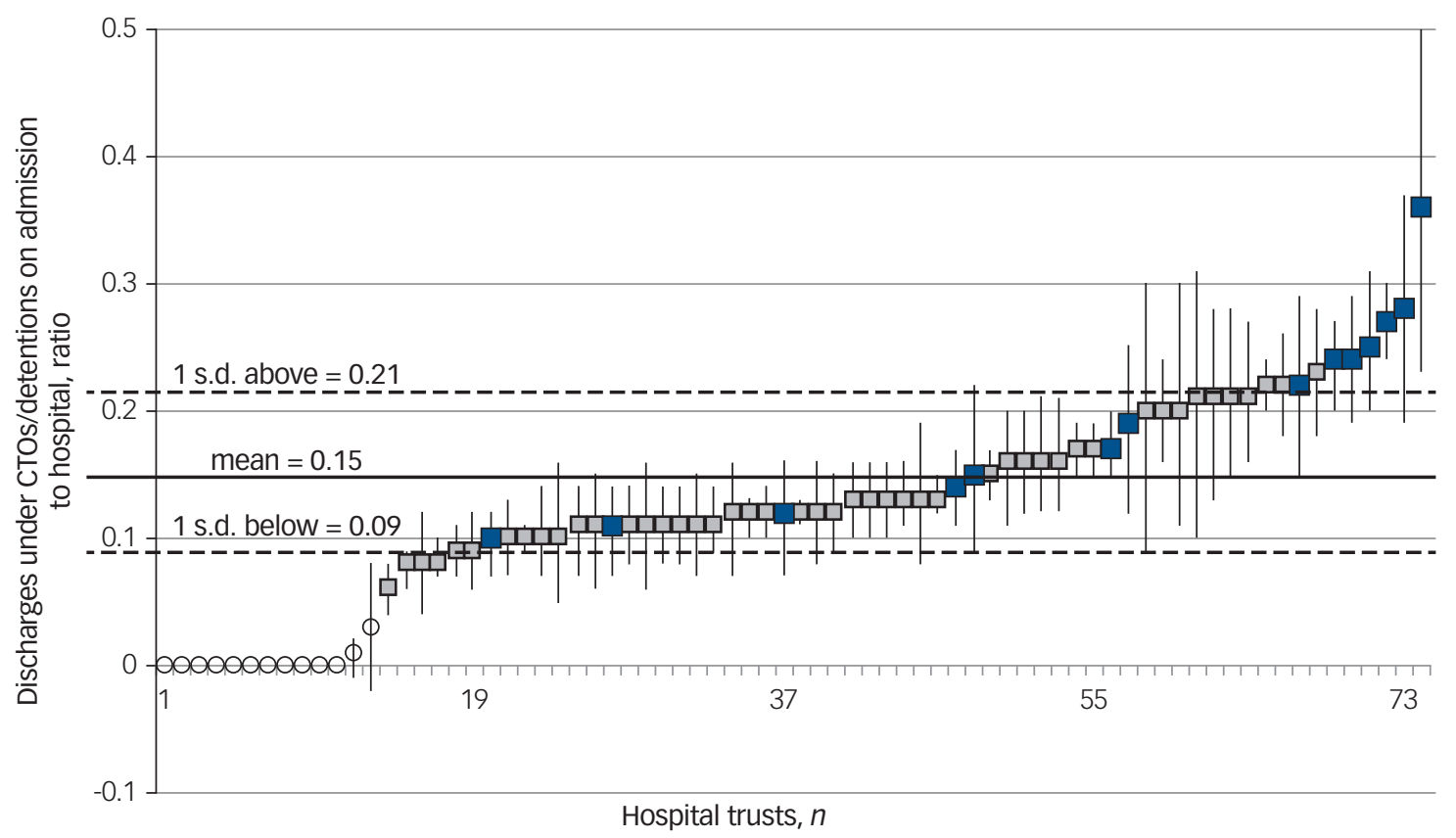

Fig. 1 The ratio of discharges under community treatment orders (CTOS) to the number of detentions on admission to hospital.

Data is displayed for 74 National Health Service hospital trusts in England. Trusts mainly providing medical/surgical/paediatric/maternity care are shown in white circles. Trust providing mainly psychiatric and learning disability services are shown in blue or grey boxes - blue boxes for hospital trusts in major urban areas, grey boxes for hospital trust outside major urban areas. The mean ratio (solid line) and standard deviation (dashed line) for mental illness and learning disability services are also shown. 
shown as blue boxes in Fig. 1. The mean ratio in urban areas was 0.20 (95\% CI $0.16-0.25, n=14$ ) or one new CTO for every five detentions on admission. This compared with an average of 0.14 (95\% CI $0.13-0.15, n=47$ ) or one new CTO for every seven detentions on admission in other trusts.

\section{Discussion}

\section{Main findings}

This paper describes the first 5 years of the use of CTOs following their introduction in England. The number of new CTOs per year has been higher than anticipated by some ${ }^{19}$ but appears to have stabilised at around 4000 per year. The prevalence rate of people who are subject to CTOs on the 31 March each year steadily increased during the first 5 years of their use, and by the end of the study period the rate was 10.0 per 100000 .

The number of new CTOs each year was substantially lower than the number of detentions in hospitals in England, indicating that only a subset of detained patients are subsequently discharged on CTOs. Our results lead us to estimate that just under $10 \%$ of individuals who are detained in hospital in a year are subsequently discharged on a CTO, and this increases to just under $20 \%$ if only those detained on longer-term sections ( $>28$ days) are considered, and increases further to just under $50 \%$ if only those on eligible forensic sections are considered.

There was a slight rise in the yearly number of new CTOs during the study period, but there was also an increase in other detentions under the Mental Health Act. However, there was a much greater increase in the prevalence rate of individuals who were subject to CTOs on the 31 March each year. These results indicate that on average each CTO was lasting longer than a year. Data from subsequent years indicates that the prevalence rate has now stabilised at around 10 per $100000 .^{20}$

In this paper we demonstrate a consistent relationship between the number of new CTOs with the number of detentions in hospital each year. In particular this relationship was evident for the number of detentions that happened at the time of admission to hospital. This is highly relevant as the main power provided by CTOs is the power of recall to hospital. The intention was that this would enable clinical teams to intervene early when a patient with a history of previous detentions is relapsing, and if appropriate prior to when the threshold for a new involuntary detention is met.

\section{The use of CTOs following civil detention}

The majority (greater than 90\%) of CTOs followed on from detention under a civil detention, and there was one new CTO for every six of these detentions. Further research is required to identify the characteristics of those patients who are discharged as voluntary patients following a period of detention compared with those who are discharged on a CTO.

\section{The use of CTOs following forensic detention}

A minority of CTOs followed on from detention under a forensic detention. This was for two reasons. First, there were far fewer forensic detentions than civil detentions each year. Second, CTOs could only be used following on from certain forensic detentions and in particular only those that did not involve a restriction order. During the study period the proportion of forensic detentions without restriction orders fell. There was one new CTO for every two eligible forensic detentions and the data we have indicates that the proportion of forensic detentions that were discharged on CTOs increased in the first 5 years.
The annual figures on detentions under the Mental Health Act suggest that prior to CTOs roughly just over a third of patients were eventually discharged from forensic detention as a voluntary patient. Our results indicate that this proportion has fallen to less than $15 \%$. This may reflect an increasing focus on managing risk when patients are discharged. The increasing use of CTOs in forensic services may reflect that following their introduction clinicians start finding them helpful and so use them more frequently. Alternatively, clinicians may feel exposed if they do not use this option when planning discharges when there are ongoing risks. Certainly, there is a need for research to focus on the function of CTOs within forensic services. Given the paucity of empirical evidence in this area (we could not identify any relevant international studies at the time of publishing), there is a need for further research to assess the efficacy of CTOs for patients being discharged from forensic sections of the Mental Health Act.

\section{Variation in the use of CTOs in NHS trusts}

Detentions on admission occurred in the majority of NHS hospital trusts. Some patients are detained to NHS hospital trusts that mainly provide medical and surgical services, for example following an overdose. However, if they required a longer period of detention they are usually transferred to a NHS hospital trust that mainly provides mental health and learning disability services. This is the reason that only those NHS hospital trusts with mental health and learning disability services discharged patients on CTOs.

There was a sixfold variation in the rate at which CTOs were used compared with the rate of detentions on admission in mental health and learning disability NHS hospital trusts. This raises important questions. It is already known that rates of detention vary significantly between trusts in major urban areas compared with those in rural areas. ${ }^{16}$ In major urban areas the population was much younger and this was part of the explanation for the higher rates of detention. ${ }^{16}$ Similarly evidence from other jurisdictions indicates that involuntary community treatment is more often used in adults of working age, with an average age between 36 and 41 years. ${ }^{2}$ In addition there is a wide range of views between psychiatrists about the use of CTOs that is likely to be reflected in differences in the use of CTOs in practice between these clinicians. ${ }^{21}$

Another possible explanation is that there may be considerable variation between trusts and between clinicians within trusts in the use of CTOs. Given that clinicians have negative views about the administrative burden of CTOs, which add to the already substantial workload of community staff, it may be that CTOs are more commonly used in specialist teams such as assertive outreach and learning disability teams who can perhaps more easily absorb the additional work. ${ }^{22}$ The variation in CTO use between teams will be worthy of further investigation.

Alternatively, it may be that the clinical and demographic characteristics associated with the use of CTOs vary between the catchment areas of different trusts. A third explanation is that the threshold for detention on admission may vary between trusts.

\section{Implications}

An important finding from this study is that there appears to be a link between the number of detentions in hospital and the number of CTOs. This suggests that if the rate of detention continues to rise then so will the use of CTOs. Conversely if the rate of detention can be reduced then the use of CTOs may fall. In this regard access to mental illness and learning disability beds will be important. ${ }^{23}$ Ensuring an adequate provision of inpatient beds to enable timely admission, on a voluntary basis where appropriate, may avoid the need for detention under the MHA at a later stage. Furthermore, there has been a recent ideologically driven 
closure of learning disability beds and this may also have an impact on future rates of CTO use.

Liam Trevithick, BSc, Tees Esk and Wear Valleys National Health Service (NHS) Foundation Trust, Darlington; Jane Carlile, MRCPsych, Northumberland Tyne and Wear NHS Foundation Trust, Newcastle; Sunil Nodiyal, MD, Patrick Keown, MRCPsych, Northumberland Tyne and Wear NHS Foundation Trust and Institute of Neuroscience, Newcastle University, Newcastle, UK

Correspondence: Patrick Keown, Tranwell Unit, Windy Nook Rd, Gateshead NE10 9RW. Email: patrick.keown@newcastle.ac.uk

First received 20 Dec 2016, final revision 30 Oct 2017, accepted 20 Nov 2017

\section{References}

1 Geller JL. The last half-century of psychiatric services as reflected in psychiatric services. Psychiatr Serv 2000; 51: 41-67.

2 Churchill R, Owen G, Singh S, Hotopf M. International Experiences of Using Community Treatment Orders. Department of Health, London, 2007.

3 Department of Health. Mental Health Bill: Regulatory Impact Assessment Revised Version. Department of Health, 2007.

4 Burgess P, Bindman J, Leese M, Henderson C, Szmukler G. Do community treatment orders for mental illness reduce readmission to hospital? SoC Psychiatry Psychiatr Epidemiol 2006; 41: 574-9.

5 New York State Office of Mental Health. Kendra's Law: Final Report on the Status of Assisted Outpatient Treatment AOT Program Administration. New York State Office of Mental Health, 2005.

6 Swartz MS, Swanson JW, Wagner HR, Burns BJ, Hiday VA, Borum R. Can involuntary outpatient commitment reduce hospital recidivism?: findings from a randomized trial with severely mentally ill individuals. Am J Psychiatry 1999; 156: $1968-75$

7 Steadman HJ, Gounis K, Dennis D, Hopper K, Roche B, Swartz M, et al. Assessing the New York City involuntary outpatient commitment pilot program. Psychiatr Serv 2001; 52: 330-6.

8 Munetz MR, Galon PA, Frese FJ III. The ethics of mandatory community treatment. J Am Acad Psychiatry Law 2003; 31: 173-83.
9 Snow N, Austin WJ. Community treatment orders: the ethical balancing act in community mental health. J Psychiatr Ment Health Nurs 2009; 16: 177-86.

10 Lawton-Smith S. A Question of Numbers. The Potential Impact of CommunityBased Treatment Orders in England and Wales. King's Fund, 2005.

11 Department of Health. Legal Powers on the Care of Mentally III People in the Community. Report of the Internal Review. Department of Health, 1993.

12 Lawton-Smith S. Supervised Community Treatment, Briefing Paper 2. Mental Health Alliance, 2010.

13 Burns T, Rugkåsa J, Molodynski A, Dawson J, Yeeles K, Vazquez-Montes M, et al. Community treatment orders for patients with psychosis (OCTET): a randomised controlled trial. Lancet 2013; 381: 1627-33.

14 Burns T, Yeeles K, Koshiaris C, Vazquez-Montes M, Molodynski A, Puntis S, et al. Effect of increased compulsion on readmission to hospital or disengagement from community services for patients with psychosis: follow-up of a cohort from the OCTET trial. Lancet Psychiatry. 2015; 312: 881-90.

15 Kisely SR, Campbell LA, O'Reilly R. Compulsory community and involuntary outpatient treatment for people with severe mental disorders. Cochrane Database Syst Rev 2017; 3: CD004408.

16 Keown P, McBride O, Twigg L, Crepaz-Keay D, Cyhlarova E, Parsons H, et al. Rates of voluntary and compulsory psychiatric in-patient treatment in England: an ecological study investigating associations with deprivation and demographics. Br J Psychiatry 2016; 209: 157-61.

17 Light E, Kerridge I, Ryan C, Robertson M. Community treatment orders in Australia: rates and patterns of use. Australas Psychiatry 2012; 20: 478-82.

18 Office for National Statistics. National Population Projections: 2014-based Statistical Bulletin. Office for National Statistics, 2015.

19 Care Quality Commission. Annual Reports and Accounts 2010/11. Care Quality Commission, 2011.

20 Community and mental health team, NHS Digital. Inpatients Formally Detained in Hospitals Under the Mental Health Act 1983, and Patients Subject to Supervised Community Treatment. NHS Digital, 2016.

21 Manning C, Molodynski A, Rugkåsa J, Dawson J, Burns T. Community treatment orders in England and Wales: national survey of clinicians' views and use. Psychiatrist 2011; 35: 328-33.

22 DeRidder R, Molodynski A, Manning C, McCusker P, Rugkåsa J. Community treatment orders in the UK 5 years on: a repeat national survey of psychiatrists. BJPsych Bull 2016; 40: 119-23.

23 Keown $\mathrm{P}$, Weich S, Bhui KS, Scott J. Association between provision of mental illness beds and rate of involuntary admissions in the NHS in England 19882008: ecological study. BMJ 2011; 343: d3736.

\section{Appendix}

\begin{tabular}{|c|c|c|}
\hline Trust & Year & Suspected data error \\
\hline Great Western Hospitals Trust & 2009-2010 & $\begin{array}{l}38 \text { formal admissions; } 104 \text { CTOs. In all other trusts the number of formal } \\
\text { admissions were much higher than number of CTOs. }\end{array}$ \\
\hline Birmingham and Solihull Mental Health Foundation Trust & $2011-2012$ & $\begin{array}{l}779 \text { formal admissions, } 0 \text { CTOs. The return of zero CTOs may be an error as in } \\
\text { other years this trust had more than } 150 \text { CTOs. }\end{array}$ \\
\hline Cornwall Partnership Trust & 2012-2013 & $\begin{array}{l}262 \text { formal admissions, } 0 \text { CTOs. The return of zero CTOs may be an error as in } \\
\text { other years this trust had more than } 20 \text { CTOs. }\end{array}$ \\
\hline
\end{tabular}

